\title{
Autonomic Behaviors of Swarm Robots Driven by Emotion and Curiosity
}

\author{
Takashi Kuremoto $^{1}$, Masanao Obayashi ${ }^{1}$, Kunikazu Kobayashi $^{1}$, Liang-Bing Feng ${ }^{1}$ \\ ${ }^{1}$ Graduate School of Science and Engineering, Yamaguchi University \\ 755-8611 Tokiwadai 2-16-1, Ube, Yamaguchi, Japan \\ \{wu, m.obayas, koba, n007we\}@yamaguchi-u.ac.jp
}

\begin{abstract}
This paper proposes an improved internal model with emotional and curious factors for autonomous robots. Robots acquire adaptive behaviors in the unknown environment according to make observation of behaviors of others. Cooperative relation among the robots and transition of curiosity to the local environments drive robots to achieve the goal of the environment exploration. Simulations showed the effectiveness of the proposed model with interesting motions of robots.
\end{abstract}

Keywords: autonomous robot, swarm robots, emotion, curiosity.

\section{Introduction}

Autonomic behaviors of swarm robots make an important role in the adaptability of robots to the complex and dynamical environments. An autonomous robot may acquire valuable information by observing the behaviors of other robots, and also be able to provide own information to others [1-4]. Meanwhile, the factors of emotion and curiosity are considered to concern with the level of intelligence of lives, i.e., more complicated mental states which come from more emotional experiences [5] symbolizes higher intelligence.

Recently, a group of Ide and Nozawa proposed an emotion model which drives autonomous robots avoiding obstacles and exploring a goal in the unknown environments [3] and [4]. The model is based on Russell's "circumplex model of affect" which assigns 8 kinds of major emotions on a 2-dimensional map [5] and [6]. Using psychological analysis of evidences, Russell categorized affective states such as pleasure, excitement, arousal, distress, misery, depression, sleepiness, and contentment orderly in a pleasure-arousal space. The emotion model of robots given by [3] used the factors of "pleasure" and "arousal" to set a series of behavior rules for the autonomous robots. When obstacles or other robots appear in the vision of a robot, for example, the behavior rules make a reducing of the value of "pleasure" or "arousal" and an increasing of the value of "displeasure" or "sleepiness". In [4], a useful application of the emotion models to the emergence of cooperative behaviors of robots was challenged and it suggests that using the emotion model autonomous robots may possess cooperation ability in uninhabited environment such as space or deep-sea. 
However, there are several practical problems exist in the model of Ide and Nozawa: 1) The limitation of the depth of view is necessary, as it determines the information of input and effects the output of the model; 2) The restriction on speed of actions is necessary; 3) Inductive function, i.e., pleasure renewing equation has a tendency to "displeasure" more easily, and this causes all robots drop to the state of "sleepiness" easily.

In this paper, we intend to overcome the problems above, and adopt a new mental factor "curiosity" to raise the motivation of autonomous behaviors. The improved internal model is confirmed its effectiveness by kinds of comparative simulations dealing with goal-exploration problems.

\section{An Improved Internal Model of Autonomous Robot}

The main difference from traditional psychological analyses of affect is that only pleasure and arousal dimensions are stressed in the model meanwhile conventionally a set of dimensions such as displeasure, distress, depression, excitement and so on were considered independently. According to 28 stimulus words presented to 36 young peoples, [5] described the emotion categories in the circular ordering. The group of Ide and Nozawa used the concept of the circumplex model to design an emotion model to evoke interactions or cooperative behaviors of multiple autonomous robots. Furthermore, Oudeyer and Kaplan used a curiosity model which considered the influence of time factor to raise the motivation of adaptive activities of robots [7]. In this Section, the emotion model of robots is introduced at first, an improved internal model including the emotional concept and a novel calculation method of curiosity is proposed.

\subsection{A Conventional Emotion Model for Robots}

In a goal-exploration problem, robots move to search the goal and avoiding to obstacles or other robots in the unknown environments. In the conventional emotion model [3], information of local environment around the robot is obtained by the observation, and the information determines the degree of emotional vectors: "pleasure" and "arousal", which cause the motion of robot. A set of behavior rules of each robot are set as the following: 1) Local information is obtained within the environment of vision; 2) The degree of arousal is proportion to the depth of vision; 3 ) A robot comes up to the robots which are "pleasure" appearing within the vision, and comes off to ones in the opposite case; 4) A robot comes up to other robots when it is in the state of "pleasure", and comes off to the others in the opposite case; 5) The degree of pleasure is reduced when obstacles or other robots are observed, and increased in the opposite case; 6) The degree of arousal is increased when other robots are observed, and reduced in the opposite case.

Descriptions of these rules can be expressed in the following equations:

$$
\boldsymbol{R}_{i}(\boldsymbol{t}+1)=\boldsymbol{R}_{i}(\boldsymbol{t})+\boldsymbol{V}_{i}(\boldsymbol{t})
$$


Where

$$
\begin{aligned}
& P v_{j i}=\frac{P v_{j} \cdot r_{j i}}{\left|\boldsymbol{r}_{j i}\right|} \\
& \boldsymbol{P} v_{i j}=\frac{\boldsymbol{P} v_{i} \cdot \boldsymbol{r}_{i j}}{\left|\boldsymbol{r}_{i j}\right|} \\
& \boldsymbol{V}_{\boldsymbol{i}}(\boldsymbol{t}+1)=\boldsymbol{V}_{\boldsymbol{i}}(\boldsymbol{t})-\boldsymbol{l}_{1} \sum_{\boldsymbol{j}} \boldsymbol{P} \boldsymbol{v}_{j i}+\boldsymbol{l}_{2} \sum_{\boldsymbol{i}} \boldsymbol{P} \boldsymbol{v}_{\boldsymbol{i}} \\
& P v(t+1)=P v(t)+e_{p} \cdot R_{P v} \cdot P v(t) \\
& \boldsymbol{e}_{\boldsymbol{p}}=\left\{\begin{array}{lll}
-1 & (\text { where } & \left.\boldsymbol{d}_{0}>\boldsymbol{D}, \boldsymbol{d}_{\boldsymbol{r}}>\boldsymbol{D}\right) \\
1 & (\text { where } & \left.\boldsymbol{d}_{0} \leq D, \boldsymbol{d}_{\boldsymbol{r}} \leq \boldsymbol{D}\right)
\end{array}\right. \\
& A v(t+1)=A v(t)+e_{a} \cdot R_{A v} \cdot A v(t) \\
& \boldsymbol{e}_{a}=\left\{\begin{array}{llr}
-1 & (\text { where } & \left.\boldsymbol{d}_{\boldsymbol{r}}>\boldsymbol{D}\right) \\
1 & (\text { where } & \left.\boldsymbol{d}_{\boldsymbol{r}} \leq \boldsymbol{D}\right)
\end{array}\right. \\
& \boldsymbol{D}=\alpha \cdot \boldsymbol{A} \boldsymbol{v}+\boldsymbol{K}
\end{aligned}
$$

$t$ : step (time)

$\boldsymbol{R}_{i}(t)$ : position vector of robot $i$ at time $t$;

$\boldsymbol{V}_{i}(\boldsymbol{t})$ : velocity vector of robot $i$ at time $t$;

$P v_{j i}$ : influence from robot $j$ to robot $i$;

$\boldsymbol{P} \boldsymbol{v}_{i j}:$ Influence from robot $i$ to $\operatorname{robot} j$;

$\boldsymbol{r}_{i j}, \boldsymbol{r}_{j i}$ : distance vector between robot $i$ and $j$;

$\boldsymbol{l}_{1}, \boldsymbol{l}_{2}$ : emotional influence parameter;

$\boldsymbol{R}_{p v}: \quad$ rate of the change of pleasure $\left(0 \leq \boldsymbol{R}_{\boldsymbol{p} v} \leq 1\right)$;

$\boldsymbol{R}_{A v}:$ rate of the change of arousal $\left(0 \leq \boldsymbol{R}_{A v} \leq 1\right)$;

$\boldsymbol{P v}$ : degree of pleasure;

$A v$ : degree of arousal;

$\boldsymbol{d}_{\boldsymbol{o}}$ : distance from robot $i$ to the nearest obstacle;

$\boldsymbol{d}_{\boldsymbol{r}}$ : distance from robot $i$ to the nearest robot;

$D$ : depth of the vision

$\alpha, \boldsymbol{e}_{a}, \boldsymbol{e}_{p}:$ positive coefficients;

$K$ : bias of the vision.

According to these rules, patterns of behaviors of robots appear as the cases of: 1) Robots in the state of pleasure attract each other and come up to each other closely; 2) A robot in the state of pleasure moves to a direction and causes others to follow it; 3 ) Robots in the state of displeasure go away from each other. 


\subsection{An Improved Internal Model for Autonomous Robots}

Using the conventional model described in the Section 2.1, we performed simulation experiments and observed kinds of results such as robots successfully attracted each other, avoided to obstacles and achieved at the goal(s) of exploration, or failed to attract each other, or failed to achieve on the multiple goal areas in a complicated environment.

The reasons of the failed cases may be considered as: 1) Bias of the vision was set inadequately. Too large value of $\boldsymbol{K}$ caused the internal state of robot dropped into "displeasure" easily; 2) The time that robots influent each other was too short because of too high velocity; 3) There was a trend that the degree of pleasure reduced more easily than increased; 4) Low degree of the pleasure of all robots caused low degree of the arousal of robots, and the case resulted in all robots dropped into the state of sleepiness, the behaviors of exploration disappeared.

To overcome these problems and to raise the motivation of exploration, here we propose to add new rules into the emotion model and adopt a new mental factor "curiosity" into the calculation of the velocity vector as following: 1) Limit bounds of the depth of vision: $K_{\min }<K<K_{\max }$; 2) Limit a maximum value of velocity: $V_{i}(\mathrm{t})<$ $V_{\text {max }}$; 3) Make the change of emotion factor "pleasure" to be dynamical, i.e., using Eq. (10) and Eq. (11) instead of Eq. (5).

$$
\begin{array}{r}
\boldsymbol{P} \boldsymbol{v}(\boldsymbol{t}+1)=\boldsymbol{N} \cdot \frac{1}{1+\exp (-\beta \boldsymbol{x}(\boldsymbol{t}))} \\
\boldsymbol{x}(\boldsymbol{t})=\mu \sin \left(\pi\left(\boldsymbol{P v}(\boldsymbol{t})+\boldsymbol{e}_{p} \boldsymbol{M}\right)\right)
\end{array}
$$

Where $\mu, \boldsymbol{M}, \boldsymbol{N}, \beta$ are positive parameters.

4) "curiosity" means 2 situations concerning with the change of the internal state of robots: i) Robot $i$ keeps to search the goals $k(k=1,2, \ldots, k, \ldots, \mathrm{K})$ before it arrives at them and after it arrives at one goal $k$ then its "curiosity" to the goal is reduced eventually; ii) During robot $i$ exploring the environment, when it crushes to obstacles its "curiosity" is reduced eventually. Eq. (12) defines the "curiosity" and Eq. (13) builds an improved internal model of autonomous robots:

$$
\begin{gathered}
C v_{i k}(t+1)=\left\{\begin{array}{lll}
I_{k}-\lambda_{1} C v_{i k}(t) & (\text { if } & \text { goal }=1,2, k) \\
I_{k}-\lambda_{2} C v_{i k}(t) & (\text { if } & \text { obstacle } \text { exists }) \\
0 & (\text { otherwise })
\end{array}\right. \\
V_{i}(t+1)=V_{i}(t)-l_{1} \sum_{j} P v_{j i}+l_{2} \sum_{i} P v_{i j}+l_{3} \sum_{i} C v_{i k}
\end{gathered} .
$$

Where $\boldsymbol{C} \boldsymbol{v}(\boldsymbol{t})$ is a factor of "curiosity" in the improved internal model, $\boldsymbol{I}_{\boldsymbol{k}}$ are positive parameters for different goal $\boldsymbol{k}$, and coefficients $\lambda_{1}, \lambda_{2}, \boldsymbol{l}_{3}>0$. 


\section{Simulation Experiment}

To exam the internal model proposed in Section 2.2, computer simulation experiments were performed using two kinds of environments for multiple robots exploration. In a simple environment with single goal as same as in [3], the behavior of two autonomous robots was observed few differences between conventional method and the novel system, however, in a complicated environment (maze-like) with multiple goals, robots arrived at all goals only in the case of the proposed method (results of the later case is reported in Section 3.1).

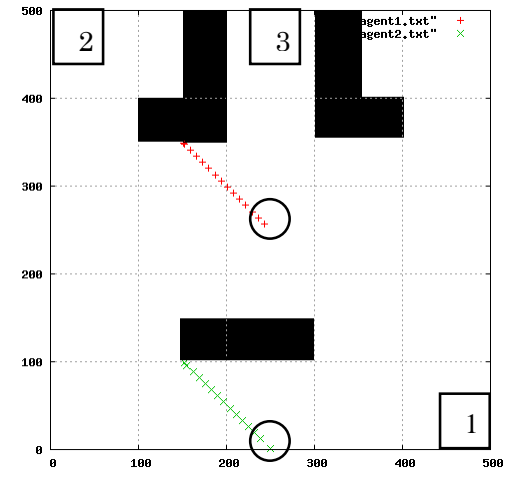

(a) Started at the same time (conventional model).

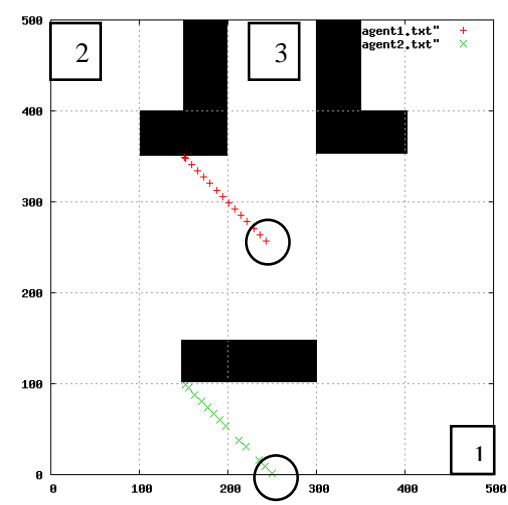

(c) Started at the different time (conventional model).

$$
\text { Start place : } \bigcirc \text { Obstacle : } \square \text { Goal : }
$$

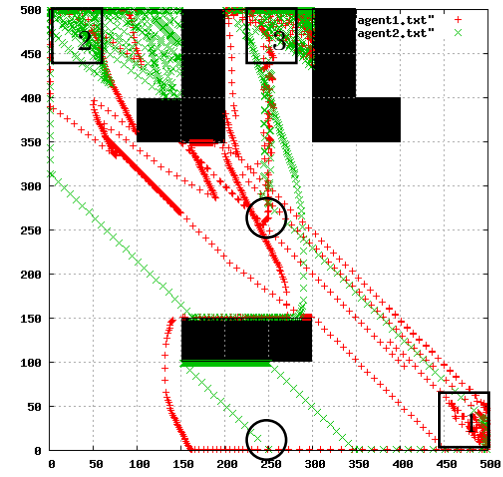

(b) Started at the same time (improved model).

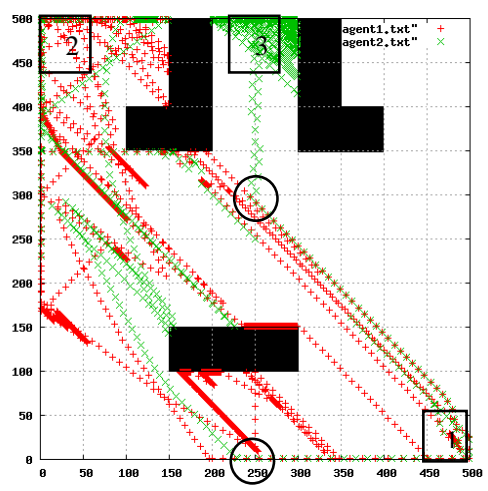

(d) Started at the different time (improved model).

Fig. 1. Tracks of 2 robots started at the same time ((a) and (b)), and different time ((c) and (d)) exploring 3 goals in a complicated unknown environment (size of the environment: 500x500 steps). 


\subsection{Finding Multiple Goals in Complicated Environment}

The size of a 2-dimensional exploring space is $500 \times 500$ (steps), multiple obstacles exist in the different positions of the square, 2 robots start from 2 different positions to search 3 goal areas located at the different positions: the environment of simulation is shown in Fig. 2. Two cases of timing of start of robots were executed: i) 2 robots started at the same time; ii) one started 200 steps later. All parameters were set as shown in Table 1, and the limitation of steps of a trial was set to 2,000 steps.

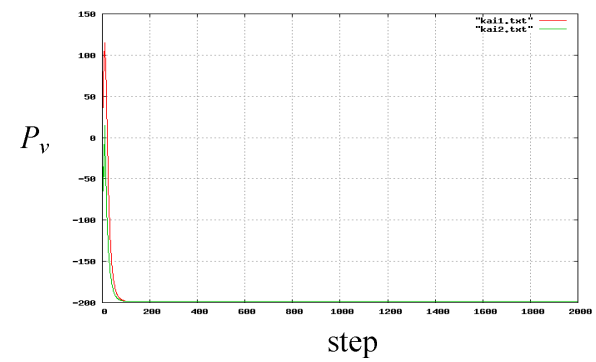

(a) Started at the same time (conventional model).

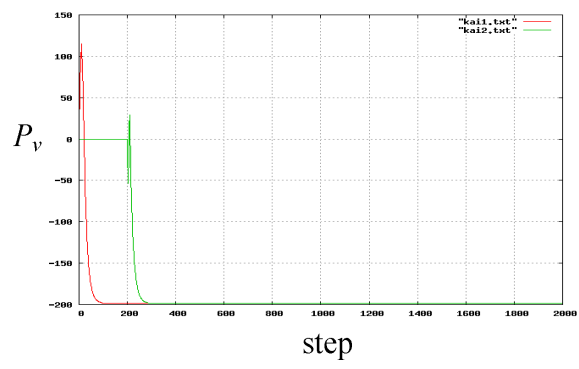

(c) Started at the different time (conventional model).

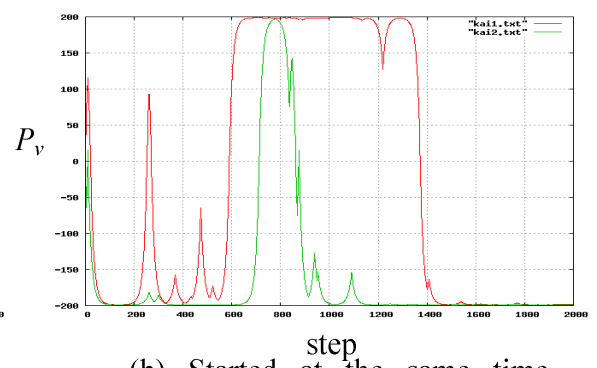

(b) Started at the same time (improved model).

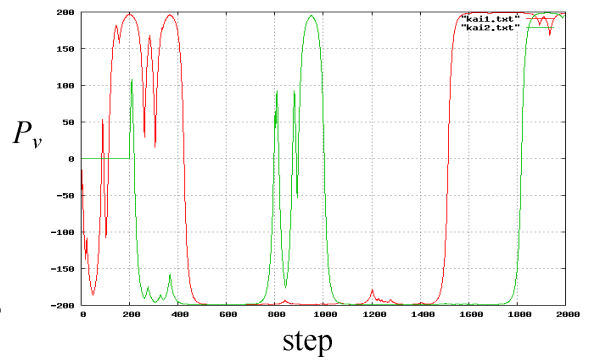

(d) Started at the different time (improved model).

Fig. 2. Comparison of the change of pleasure degrees of 2 robots exploring 3 goals in the complicated unknown environment.

i) Simulation results of 2 robots started at the same time are shown in Fig. 1 (a) and Fig. 1 (b). Robots with conventional model stopped exploration for the kinds of reasons such as obstacles and multiple goals (Fig. 1 (a)), meanwhile, those with the improved model showed active exploration and reached at all 3 goals (Fig. 1 (b)).

ii) Simulation results of 2 robots started at the different time are shown in Fig. 1 (c) and Fig. 1 (d). Robots with conventional model also stopped exploration without reaching to any goal (Fig. 1 (c)). Robots with the improved model also showed active exploration, however, one failed to reach at Goal 3 (Fig. 1 (d)).

The change of the degree of pleasure, as curves depicted in Fig. 2 (a)-(d) respective to Fig. 1 (a)-(d), showed the difference of the internal state changing of 2 robots between conventional model and improved model. More dynamical activity was observed in the case of our model. 
Table 1. Parameters used in the simulation of this section.

\begin{tabular}{lll}
\hline Parameter & Symbol & Value \\
\hline The value of pleasure at start and goals & $\boldsymbol{P} \boldsymbol{v}(0), \boldsymbol{P} \boldsymbol{v}$ & $0.0,1,200.0$ \\
Coefficients of emotional influence factors & $\boldsymbol{l}_{1}, \boldsymbol{l}_{2}$ & $6.0,1.0$ \\
Coefficient of curiosity influence factor & $\boldsymbol{l}_{3}$ & 0.5 \\
Bounds of the vision & $K_{\min }, K_{\max }$ & $30.0,50.0$ \\
Limitation to velocity & $V_{\max }$ & 15.0 \\
Parameters in dynamical pleasure calculation & $\boldsymbol{N}, \boldsymbol{M}, \mu, \beta$ & $100,100,100,0.05$ \\
Coefficients in curiosity calculation & $\lambda_{1}, \lambda_{2}$ & $0.2,0.2$ \\
Initial value of curiosity & $\boldsymbol{C}_{v}$ & 0.0 \\
The value of curiosity for goal area & $\boldsymbol{I}_{\boldsymbol{k}}$ & 20.0 \\
\hline
\end{tabular}

\section{Conclusion}

An emotion-curiosity driven behavior model is proposed for the exploration activity of swarm robots. The basic idea of the internal model is that metal states including pleasure, arousal and curiosity motivate robots control the velocities in time. Simulations showed the effectiveness of the proposed model. This research suggests that the mental models may serve important roles in the art of swarm robot design.

Acknowledgements. We would like to thank Y. Matsusaki and M. Sugino for their early work, and a part of this study was supported by JSPS-KAKENHI (No.20500207 and No.20500277).

\section{References}

1. Cao, Y. U., Fukunaga, A. S., Kahng, A. B.: Cooperative Mobile Robotics: Antecedents and Directions. Autonomous Robots, vol.4, pp.7--27 (1997)

2. Asada, M., Uchibe, E., Hosoda, K.: Cooperative behavior acquisition for mobile robots in dynamically changing real worlds via vision-based reinforcement learning and development. Artificial Intelligence, vol. 110, pp.275--292 (1999)

3. Sato, S., Nozawa, A., and Ide, H.: Characteristics of Behavior of Robots with Emotion Model. IEEJ Trans. EIS, vol. 124, no. 7, pp. 1390--1395 (2004) (in Japanese)

4. Kusano, T., Nozawa, A., and Ide, H.: Emergent of Burden Sharing of Robots with Emotion Model. IEEJ Trans. EIS, vol. 125, no. 7, pp. 1037--1042 (2005) (in Japanese)

5. Russell, J. A.: A circumplex model of affect. Journal of Personality and Social Psychology, vol.39, No.6, pp. 1161--1178 (1980)

6. Larsen, R. J., Diener, E.: Promises and problems with the circumplex model of emotion. In M.S. Clark (Ed.), Review of Personality and Social Psychology:Emotion, vol.13, pp. 25--59, Newbury Park, CA: Sage (1992)

7. Oudeyer, P. -Y., Kaplan, F.: Intelligent Adaptive Curiosity: a Source of Self-Development. In Proc. $4^{\text {th }}$ Intern. Workshop on Epigenetic Robotics, pp. 12--132 (2004) 